\title{
Addendum to 'Perelman's reduced volume and a gap theorem for the Ricci flow'
}

\author{
TAKUMI YOKOTA
}

\begin{abstract}
In this addendum to [10], we prove that if the Gaussian density of a complete gradient shrinking Ricci soliton is close to that of the Gaussian soliton, then they are isometric to each other. This was shown in [10] under the additional assumption that its Ricci curvature is bounded below. We drop this assumption by developing Perelman's reduced geometry for arbitrary complete gradient shrinking Ricci solitons.
\end{abstract}

\section{Background}

In [10], we dealt with ancient solutions to the Ricci flow equation and gradient shrinking Ricci solitons. A one-parameter family of Riemannian metrics $(M, g(t)), t \in I \subset \mathbb{R}$ on a manifold $M$ is called a Ricci flow when it evolves along the equation

$$
\frac{\partial}{\partial t} g(t)=-2 \operatorname{Ric}(g(t))
$$

where $\operatorname{Ric}(g(t))$ denotes the Ricci tensor of $g(t)$, and it is called an ancient solution if it exists for all $t \in(-\infty, 0]$. It is convenient to use the reverse time parameter $\tau:=-t$ to handle ancient solutions.

A triple $(M, g, f)$ consisting of a manifold $M$ with a Riemannian metric $g$ and a smooth function $f \in C^{\infty}(M)$ is called a gradient shrinking Ricci soliton if it satisfies the following identity for some positive constant $\lambda>0$ :

$$
\operatorname{Ric}(g)+\operatorname{Hess} f=\frac{1}{2 \lambda} g \text {. }
$$

We always normalize the potential function $f$ by adding a constant so that

$$
\lambda\left(R+|\nabla f|^{2}\right)=f \text { on } M .
$$

We called $\operatorname{Vol}_{f}(M):=\int_{M}(4 \pi \lambda)^{-n / 2} \mathrm{e}^{-f} d \mu_{g}$ the normalized $f$-volume of $\left(M^{n}, g, f\right)$ in [10], while it was called the Gaussian density in [1]. Here $R$ 
and $d \mu_{g}$ denote the scalar curvature and the volume element of $g$, respectively. Shrinking Ricci solitons are typical examples of ancient solutions to the Ricci flow.

The purpose of this addendum is to prove the following general gap theorem for gradient shrinking Ricci solitons.

Theorem 2 (cf. [10, Corollary 1.1]). There exists a constant $\varepsilon_{n}>0$ which depends only on $n \geq 2$ and satisfies the following: Any complete $n$-dimensional gradient shrinking Ricci soliton $\left(M^{n}, g, f\right)$ with $\operatorname{Vol}_{f}(M)>$ $1-\varepsilon_{n}$ is, up to scaling, the Gaussian soliton $\left(\mathbb{R}^{n}, g_{E}, \frac{|\cdot|^{2}}{4}\right)$.

In [10], Theorem 2 was obtained under the additional assumption that the Ricci curvature is bounded below as a corollary of [10, Theorem 1.1], which is a gap theorem for ancient solutions to the Ricci flow with Ricci curvature bounded below. In its statement, we need the assumption on the Ricci curvature to ensure that Perelman's reduced volume is well-defined. In this addendum, we observe that this is the case for ancient solutions generated by any gradient shrinking Ricci solitons. Then our Theorem 2 follows from [10, Theorem 1.1]. We recall that, in the proof of [10, Theorem 1.1], we have never used the assumption that its Ricci curvature is bounded below (cf. [10, Remark 6.6]).

After we review the definition of Perelman's reduced volume in Section 2, we present a proof of Theorem 2 in Section 3. We conclude this section by collecting several remarks on Theorem 2.

After the appearance of the paper [10], Enders-Müller-Topping [5] applied Theorem 2 to obtain a regularity type theorem for type I Ricci flow, while Munteanu-Wang [8] obtained another interesting gap theorem for gradient shrinking Ricci solitons with pinched Ricci tensor. We also comment that one of the assumptions in Haslhofer-Müller's precompactness theorem proved in [6] for sequences of gradient shrinking Ricci solitons is a uniform positive lower bound for $\operatorname{Vol}_{f}(M)$.

We remark again that our result is intimately related to the results and conjecture by Carrillo-Ni [3] (cf. [10, Remark 6.5]); Namely, Theorem 2 gives a complete affirmative answer to their conjecture that $\operatorname{Vol}_{f}(M)=1$ for and only for the Gaussian soliton.

\section{Perelman's reduced volume}

In this section, we briefly recall the definition of Perelman's reduced volume given in $[9$, Sections 6,7$]$. We use the same notations as in [10] to which we refer for references as well. 
Definition 3. Let $\left(M^{n}, g(\tau)\right), \tau \in[0, T)$ be a backward Ricci flow, and take $p, q \in M$ and $\left[\tau_{1}, \tau_{2}\right] \subset[0, T)$. We define the $\mathcal{L}$-length of a curve $\gamma:\left[\tau_{1}, \tau_{2}\right] \rightarrow$ $M$ and the $\mathcal{L}$-distance, respectively, by

$$
\mathcal{L}^{g}(\gamma):=\int_{\tau_{1}}^{\tau_{2}} \sqrt{\tau}\left(|\dot{\gamma}(\tau)|_{g(\tau)}^{2}+R(\gamma(\tau), \tau)\right) d \tau
$$

and $L_{\left(p, \tau_{1}\right)}^{g}\left(q, \tau_{2}\right):=\inf \mathcal{L}^{g}(\gamma)$, where the infimum is taken for all curves $\gamma$ : $\left[\tau_{1}, \tau_{2}\right] \stackrel{M}{\rightarrow} M$ with $\gamma\left(\tau_{1}\right)=p$ and $\gamma\left(\tau_{2}\right)=q$. Then the reduced volume based at $(p, 0)$ is defined by

$$
\tilde{V}_{(p, 0)}^{g}(\tau):=\int_{M}(4 \pi \tau)^{-n / 2} \exp \left(-\frac{1}{2 \sqrt{\tau}} L_{(p, 0)}^{g}(\cdot, \tau)\right) d \mu_{g(\tau)},
$$

where $d \mu_{g(\tau)}$ denotes the volume element induced by $g(\tau)$.

The existence of the lower bound for $\operatorname{Ric}\left(=\frac{1}{2} \frac{\partial}{\partial \tau} g(\cdot)\right)$ guarantees that the $\mathcal{L}$-distance between any two points is achieved by a minimal $\mathcal{L}$-geodesic. Then Perelman [9] proved that the reduced volume is nonincreasing in $\tau>0$ and its value does not exceed 1 (cf. [10, Theorem 2.1]).

We comment that, in [10, Section 2], we developed Perelman's reduced geometry for more general geometric flows including the Ricci flow under somewhat artificial assumptions, and independently and simultaneously, Müller [7] also obtained almost the same result.

\section{A refinement of a gap theorem for gradient shrinkers}

In this section, we present a proof of Theorem 2. Our proof relies on the following result concerning the geometry of gradient shrinking Ricci solitons.

Theorem 4 (Cao-Zhou [2], cf. Haslhofer-Müller [6]). Let $\left(M^{n}, g, f\right)$ be a complete gradient shrinking Ricci soliton and $x_{0} \in M$ be fixed. Then

$$
\frac{1}{4}\left(r(x)-c_{1}\right)^{2} \leq f(x) \leq \frac{1}{4}\left(r(x)+c_{2}\right)^{2}
$$

for any $x \in M$ with $r(x):=d\left(x, x_{0}\right) / \sqrt{\lambda} \gg 1$ large, and

$$
\operatorname{Vol}\left(B_{x_{0}}(r)\right) \leq C r^{n} \text { for any } r>0 .
$$

Here $B_{x_{0}}(r)$ is the closed ball of radius $r>0$, and $c_{i}, i=1,2$, and $C>0$ denote some constants. 
Proof of Theorem 2 (cf. Proof of [10, Corollary 1.1]). Let $(M, g, f)$ be a complete gradient shrinking Ricci soliton with $f$ being normalized as in (1.1). We begin the proof by constructing a Ricci flow $\left(M, g_{0}(\tau)\right), \tau \in(0, \infty)$, where $g_{0}(\tau):=(\tau / \lambda)\left(\psi_{\tau}\right)^{*} g$ for the family $\left\{\psi_{\tau}:=\varphi_{\tau}^{-1}\right\}$ of diffeomorphisms of $M$ defined by

$$
\frac{d}{d \tau} \varphi_{\tau}(\cdot)=\frac{\lambda}{\tau} \nabla^{g} f\left(\varphi_{\tau}(\cdot)\right) \text { and } \varphi_{\lambda}=\operatorname{id}_{M}
$$

As was shown by Zhang [11], $\nabla^{g} f$ is complete provided $g$ is complete.

We now list several facts that will be required in the proof. At first, any complete gradient shrinking Ricci soliton have nonnegative scalar curvature $R \geq 0$ and potential function $f \geq 0$ (Zhang [11]). Next, letting $f_{\tau}:=f \circ \psi_{\tau}$, we have

$$
L_{\left(p, \tau_{1}\right)}^{g_{0}}\left(q, \tau_{2}\right) \geq 2 \sqrt{\tau_{2}} f_{\tau_{2}}(q)-2 \sqrt{\tau_{1}} f_{\tau_{1}}(p)
$$

for any $\left(p, \tau_{1}\right),\left(q, \tau_{2}\right) \in M \times(0, \infty)$ with $\tau_{2}>\tau_{1}$.

Inequality (3.3) is derived as follows (cf. [4, p. 344]): It is easy to see that

$$
\frac{\partial f_{\tau}}{\partial \tau}=-\left|\nabla^{g_{0}(\tau)} f_{\tau}\right|_{g_{0}(\tau)}^{2} \text { and } R_{g_{0}}(\cdot, \tau)+\left|\nabla^{g_{0}(\tau)} f_{\tau}\right|_{g_{0}(\tau)}^{2}=\frac{f_{\tau}}{\tau}
$$

Then, for any curve $\gamma:\left[\tau_{1}, \tau_{2}\right] \rightarrow M$, we have

$$
\begin{aligned}
\frac{d}{d \tau} 2 \sqrt{\tau} f_{\tau}(\gamma(\tau)) & =\sqrt{\tau}\left(|\dot{\gamma}(\tau)|_{g_{0}(\tau)}^{2}+R_{g_{0}}(\gamma(\tau), \tau)-\left|\dot{\gamma}-\nabla^{g_{0}(\tau)} f_{\tau}\right|_{g_{0}(\tau)}^{2}\right) \\
& \leq \sqrt{\tau}\left(|\dot{\gamma}(\tau)|_{g_{0}(\tau)}^{2}+R_{g_{0}}(\gamma(\tau), \tau)\right),
\end{aligned}
$$

and integrating this inequality yields (3.3).

Finally, we state the following sublemma. This is a restatement of $[10$, Sublemma 3.1], which played crucial role in [10].

Sublemma 8 ([10, Sublemma 3.1]). Let $\left(M, g_{0}(\tau)\right)$ be a backward Ricci flow and $\left(M, g_{1}(\tau)\right), \tau \in[0, T)$ be another backward Ricci flow with nonnegative scalar curvature $R \geq 0$ satisfying that $g_{1}(\tau):=g_{0}\left(\tau+\tau_{\Delta}\right)$ for some $\tau_{\Delta}>0$. Then, for any curves $\gamma_{1}:\left[\tau_{1}, \tau_{2}\right] \rightarrow M$ and $\gamma_{0}:\left[\tau_{1}+\tau_{\Delta}, \tau_{2}+\tau_{\Delta}\right] \rightarrow$ $M$ with $\left[\tau_{1}, \tau_{2}\right] \subset[0, T)$ and $\gamma_{1}(\cdot)=\gamma_{0}\left(\cdot+\tau_{\Delta}\right)$, we have

$$
\frac{1}{2 \sqrt{\tau_{2}+\tau_{\Delta}}} \mathcal{L}^{g_{0}}\left(\gamma_{0}\right) \geq \frac{1}{2 \sqrt{\tau_{2}}} \mathcal{L}^{g_{1}}\left(\gamma_{1}\right) \geq \alpha_{\tau_{1}, \tau_{2}}^{\tau_{\Delta}} \cdot \frac{1}{2 \sqrt{\tau_{2}+\tau_{\Delta}}} \mathcal{L}^{g_{0}}\left(\gamma_{0}\right)
$$

where $\alpha_{\tau_{1}, \tau_{2}}^{\tau_{\Delta}}:=\sqrt{\frac{\tau_{1}}{\tau_{1}+\tau_{\Delta}} \frac{\tau_{2}+\tau_{\Delta}}{\tau_{2}}}$. 
Then we put $g_{1}(\tau):=g(\tau+\lambda), \tau \in[0, \infty)$ to obtain an ancient solution $\left(M, g_{1}(\tau)\right), \tau \in[0, \infty)$. Now, our main lemma is

Lemma 10. For any $\left(p, \tau_{1}\right),\left(q, \tau_{2}\right) \in M \times[0, \infty)$ with $\tau_{2}>\tau_{1}$, there exists a minimal $\mathcal{L}^{g_{1}}$-geodesic $\gamma$ connecting $\left(p, \tau_{1}\right)$ and $\left(q, \tau_{2}\right)$, that is $\mathcal{L}^{g_{1}}(\gamma)=$ $L_{\left(p, \tau_{1}\right)}^{g_{1}}\left(q, \tau_{2}\right)$.

Proof. It suffices to prove that any minimizing sequence $\left\{\gamma_{i}\right\}$ of curves connecting $\left(p, \tau_{1}\right)$ and $\left(q, \tau_{2}\right)$ for which $\mathcal{L}^{g_{1}}\left(\gamma_{i}\right) \rightarrow L:=L_{\left(p, \tau_{1}\right)}^{g_{1}}\left(q, \tau_{2}\right)$ stays in a bounded region of $M$. It is clear that $L<\infty$. Then, by taking a converging subsequence from it, we can find a minimal $\mathcal{L}^{g_{1}}$-geodesic (e.g. [4, Lemma 7.27]).

To begin with, we take a compact subset $\mathcal{K} \subset M$ containing $p$ in its interior, and find $C \geq 0$ such that $\operatorname{Ric}\left(g_{1}(\cdot)\right) \geq-C$ on $\mathcal{K} \times\left[0, \tau_{2}\right]$. We let $r(\cdot):=d(\cdot, p) / \sqrt{\lambda}$ be the rescaled distance from $p$ with respect to the metric $g=g_{1}(0)$.

For any $L^{\prime} \in(L, \infty)$, we consider a curve $\gamma:\left[\tau_{1}, \tau_{2}\right] \rightarrow M$ with $\gamma\left(\tau_{1}\right)=p$, $\gamma\left(\tau_{2}\right)=q$ and $\mathcal{L}^{g_{1}}(\gamma)<L^{\prime}$. Then we find $\tau^{\prime}>0$ and $\rho^{\prime}>0$ such that any such curve $\gamma$ satisfies that $\gamma\left(\left[\tau_{1}, \tau^{\prime}\right]\right) \subset B_{p}\left(\rho^{\prime}\right) \subset \mathcal{K}$. This is possible because

$$
L^{\prime}>\mathcal{L}^{g_{1}}\left(\left.\gamma\right|_{\left[\tau_{1}, \tau^{\prime}\right]}\right) \geq \int_{\tau_{1}}^{\tau^{\prime}} \sqrt{\tau}|\dot{\gamma}(\tau)|_{g_{1}(\tau)}^{2} d \tau \geq \mathrm{e}^{-2 C \tau_{2}} \cdot \frac{\lambda r\left(\gamma\left(\tau^{\prime}\right)\right)^{2}}{2\left(\sqrt{\tau^{\prime}}-\sqrt{\tau_{1}}\right)}
$$

for any $\tau^{\prime}>\tau_{1}$ close to $\tau_{1}$.

Next, for any $\tau \in\left[\tau^{\prime}, \tau_{2}\right]$, with $\gamma_{0}:\left[\tau_{1}+\lambda, \tau_{2}+\lambda\right] \rightarrow M$ being the curve given by $\gamma_{0}(\cdot+\lambda):=\gamma(\cdot)$, we have

$$
\begin{aligned}
\frac{L^{\prime}}{2 \sqrt{\tau_{2}}} & >\sqrt{\frac{\tau}{\tau_{2}}} \cdot \frac{1}{2 \sqrt{\tau}} \mathcal{L}^{g_{1}}\left(\left.\gamma\right|_{\left[\tau^{\prime}, \tau\right]}\right) \\
& \geq \sqrt{\frac{\tau}{\tau_{2}}} \sqrt{\frac{\tau^{\prime}}{\tau^{\prime}+\lambda} \frac{\tau+\lambda}{\tau}} \cdot \frac{1}{2 \sqrt{\tau+\lambda}} \mathcal{L}^{g_{0}}\left(\left.\gamma_{0}\right|_{\left[\tau^{\prime}+\lambda, \tau+\lambda\right]}\right) \\
& \geq \sqrt{\frac{\tau}{\tau_{2}}} \sqrt{\frac{\tau^{\prime}}{\tau^{\prime}+\lambda} \frac{\tau+\lambda}{\tau}} \cdot\left[f_{\tau+\lambda}(\gamma(\tau))-\sqrt{\frac{\tau^{\prime}+\lambda}{\tau+\lambda}} f_{\tau^{\prime}+\lambda}\left(\gamma\left(\tau^{\prime}\right)\right)\right] \\
& \geq \frac{1}{4} \sqrt{\frac{\tau^{\prime}}{\tau_{2}}}\left(r\left(\psi_{\tau+\lambda}(\gamma(\tau))\right)-c_{1}\right)^{2}-\max _{B_{p}\left(\rho^{\prime}\right)} f \circ \psi_{\tau^{\prime}+\lambda}(\cdot) .
\end{aligned}
$$

We have used the fact that $R$ and $f$ are nonnegative, Sublemma 8 and (3.3). This means that we can find $D<\infty$ such that $\gamma\left(\left[\tau_{1}, \tau_{2}\right]\right) \subset B_{p}(D)$ for any $\gamma$ in consideration. This completes the proof of the lemma. 
Once we have proved Lemma 10, the proofs in the literature are available to see that the reduced volume $\tilde{V}_{(p, 0)}^{g_{1}}(\tau)$ is nonincreasing in $\tau>0$ and it takes value at most 1 . By repeating the proof of [10, Corollary 1.1], in which the first inequality in (3.4) played a key role, we obtain

$$
\operatorname{Vol}_{f}(M) \leq \widetilde{\mathcal{V}}\left(g_{1}\right):=\lim _{\tau \rightarrow \infty} \tilde{V}_{(p, 0)}^{g_{1}}(\tau) \in[0,1]
$$

Then, applying [10, Theorem 1.1] completes the proof of Theorem 2.

We conclude this addendum by presenting the following proposition, which asserts that the equality actually holds in (3.5).

Proposition 12 (cf. [10, Proposition 5.1]). Let $\left(M^{n}, g, f\right)$ be a complete gradient shrinking Ricci soliton. Then, with notation as in the proof of Theorem 2, we have

$$
\widetilde{\mathcal{V}}\left(g_{1}\right)=\operatorname{Vol}_{f}(M)
$$

Proof. We can prove that

$$
\widetilde{\mathcal{V}}\left(g_{1}\right) \leq \int_{M}(4 \pi \lambda)^{-n / 2} \mathrm{e}^{-\alpha f} d \mu_{g}
$$

for any positive $\alpha \in(0,1)$, in the same way as in the proof of [10, Proposition 5.1], in which the second inequality in (3.4) was the key. Then, the right hand side of (3.6) is finite due to Equations (3.1) and (3.2), and it converges to the normalized $f$-volume $\operatorname{Vol}_{f}(M)$ of $\left(M^{n}, g, f\right)$ as $\alpha \rightarrow 1$. Combined with (3.5), this completes the proof of the proposition.

\section{Acknowledgments}

This work was partly supported by Grant-in-Aid for Research Activity (start-up) no. 22840028.

\section{References}

[1] H.-D. Cao, R. Hamilton and T. Ilmanen, Gaussian densities and stability for some Ricci solitons, arXiv:math/0404165.

[2] H.-D. Cao and D. Zhou, On complete gradient shrinking Ricci solitons, J. Differential Geom. 85(2) (2010), 175-185.

[3] J. Carrillo and L. Ni, Sharp logarithmic Sobolev inequalities on gradient solitons and applications, Comm. Anal. Geom. 17(4) (2009), 721-753. 
[4] B. Chow, S.-C. Chu, D. Glickenstein, C. Guenther, J. Isenberg, T. Ivey, D. Knopf, P. Lu, F. Luo and L. Ni, The Ricci flow: Techniques and applications. Part I. Geometric aspects, Mathematical Surveys and Monographs, 135, American Mathematical Society, Providence, RI, 2007.

[5] J. Enders, R. Müller and P. Topping, On Type I singularities in Ricci flow, Comm. Anal. Geom. 19(5) (2011), 905-922.

[6] R. Haslhofer and R. Müller, A compactness theorem for complete Ricci shrinkers, Geom. Funct. Anal. 21 (2011), 1091-1116.

[7] R. Müller, Monotone volume formulas for geometric flows, J. Reine Angew. Math. 643 (2010), 39-57.

[8] O. Munteanu and M.-T. Wang, The curvature of gradient Ricci solitons, Math. Res. Lett. 18(6) (2011), 1051-1069.

[9] G. Perelman, The entropy formula for the Ricci flow and its geometric applications, arXiv:math/0211159.

[10] T. Yokota, Perelman's reduced volume and a gap theorem for the Ricci flow, Comm. Anal. Geom. 17(2) (2009), 227-263.

[11] Z.-H. Zhang, On the completeness of gradient Ricci solitons, Proc. Amer. Math. Soc. 37(8) (2009), 2755-2759.

Research Institute for Mathematical Sciences,

KYOTO UNIVERSITY,

КYОТО 606-8502, JAPAN

E-mail address: takumiy@kurims.kyoto-u.ac.jp

Received December 1, 2011 
